\title{
Search for water and life's building blocks in the Universe: An Introduction
}

\author{
Sun Kwok \\ Faculty of Science, The University of Hong Kong, Hong Kong, China \\ email: sunkwok@hku.hk
}

\begin{abstract}
Water and organics are commonly believed to be the essential ingredients for life on Earth. The development of infrared and submillimeter observational techniques has resulted in the detection of water in circumstellar envelopes, interstellar clouds, comets, asteroids, planetary satellites and the Sun. Complex organics have also been found in stellar ejecta, diffuse and molecular clouds, meteorites, interplanetary dust particles, comets and planetary satellites. In this Focus Meeting, we will discuss the origin, distribution, and detection of water and other life's building blocks both inside and outside of the Solar System. The possibility of extraterrestrial organics and water on the origin of life on Earth will also be discussed.
\end{abstract}

Keywords. astrobiology, astrochemistry

\section{Water in the Universe}

The water molecule was first discovered in space through its $6_{16}-5_{23}$ rotational transition at $22 \mathrm{GHz}$ (Cheung et al. 1969). The inverted population and resultant maser emission of this transition allowed this line to be detected even in the early days of millimeter-wave receiver technology. Because of obscuration of the Earth's atmosphere, the detection of the ground-state transition $557 \mathrm{GHz} 1_{10}-1_{01}$ of ortho-water has to wait until the development of satellite-based submillimeter telescopes. The $557 \mathrm{GHz}$ line was first detected by $S W A S$ and has been extensively observed in many astronomical sources by Odin and Herschel.

Earth is commonly believed to be habitable because it contains large volumes of liquid water in surface oceans. Water ice is also prevalent in the polar regions. Water, in its ice, liquid, and vapor forms, shapes the geology and influences climate of the Earth. By acting as an effective solvent, liquid water also assists many biological chemical reactions leading to complex living organisms.

Water ice is believed to be the most abundant condensed-phase species in the Universe. The $\mathrm{O}-\mathrm{H}$ stretching mode at $3.05 \mu \mathrm{m}$ and the $\mathrm{H}-\mathrm{O}-\mathrm{H}$ bending mode at $6.0 \mu \mathrm{m}$ of water ice is commonly observed in young stellar objects. In the Solar System, water ice is found in comets, asteroids, and of course in the polar caps of the Earth. Water is found on the Moon and liquid water used to flow on the surface of Mars. Water vapor has even been detected in the Sun (Wallace et al. 1995). The planets Uranus and Neptune, as well as outer Solar System objects such as Pluto and other Kuiper Belt Objects, are covered with water ice. Subterranean liquid water oceans are believed to exist in the moons of Jupiter (Europa and Ganymede) and Saturn (Enceladus). 


\section{Organic matter in the Milky Way and other galaxies}

The development of infrared and millimeter-wave technology has made possible the detection of $\sim 200$ gas-phase molecules in space. The detected species cover all kinds of organic molecules, including hydrocarbons (e.g., methane $\mathrm{CH}_{4}$, acetylene $\mathrm{C}_{2} \mathrm{H}_{2}$, ethylene $\mathrm{C}_{2} \mathrm{H}_{4}$ ), alcohols (e.g., methanol $\mathrm{CH}_{3} \mathrm{OH}$, ethanol $\mathrm{C}_{2} \mathrm{H}_{5} \mathrm{OH}$, vinyl alcohol $\mathrm{H}_{2} \mathrm{C}=\mathrm{CHOH}$ ), acids (e.g., formic acid $\mathrm{HCOOH}$, acetic acid $\mathrm{CH}_{3} \mathrm{COOH}$ ), aldehydes (e.g., formaldehyde $\mathrm{H}_{2} \mathrm{CO}$, acetaldehyde $\mathrm{CH}_{3} \mathrm{CHO}$, propenal $\mathrm{CH}_{2}=\mathrm{CHCHO}$, propanal $\mathrm{CH}_{3} \mathrm{CH}_{2} \mathrm{CHO}$ ), ketones (e.g., ethenone $\mathrm{H}_{2} \mathrm{C}=\mathrm{CO}$, acetone, $\mathrm{CH}_{3} \mathrm{COCH}_{3}$ ), amines (e.g., methylamine $\mathrm{CH}_{3} \mathrm{NH}_{2}$, cyanamide $\mathrm{NH}_{2} \mathrm{CN}$, formamide $\mathrm{NH}_{2} \mathrm{CHO}$ ), ethers (e.g., dimethyl ether $\mathrm{CH}_{3} \mathrm{OCH}_{3}$, ethyl methyl ether $\mathrm{CH}_{3} \mathrm{OC}_{2} \mathrm{H}_{5}$ ), etc. Because of the high spectral resolution of modern spectrometers, molecular transitions can be identified with frequency accuracy of 1 in $10^{7}$, making the astronomical detection of molecular species extremely robust.

Infrared observations have identified vibrational bands of aromatic (e.g., the $\mathrm{C}-\mathrm{H}$ stretch at $3.3 \mu \mathrm{m}$ ) and aliphatic (e.g., the $\mathrm{C}-\mathrm{H}$ strecth at $3.4 \mu \mathrm{m}$ ) compounds. The $3.3 \mu \mathrm{m}$ feature has been detected in galaxies with redshifts as high as 2 , suggesting that organic compounds were already present in the early Universe. The strengths of the 3.4 $\mu \mathrm{m}$ absorption features imply that $15 \%$ of $\mathrm{C}$ in the diffuse interstellar medium is in the form of aliphatic compounds (Dartois, these proceedings).

There are a number of unexplained spectral phenomena whose origin may be traced to organic compounds (Kwok 2013). The diffuse interstellar bands (DIB), the $217.5 \mathrm{~nm}$ absorption feature, the unidentified infrared emission (UIE) bands, and the extended red emission (ERE) are widely found in the Milky Way Galaxy and in external galaxies. The carriers of the more than 500 observed DIBs are not known but they are widely believed to be due to carbon-based molecules. This belief is strengthened by the recent identification of the 963.2 and $957.5 \mathrm{~nm}$ bands as due to $\mathrm{C}_{60}^{+}$(Campbell et al. 2015). Due to the widespread presence and strengths of the DIBs, there must be a large reservoir of organic molecules in the diffuse interstellar medium.

The broad $220 \mathrm{~nm}$ feature is widely observed in the diffuse interstellar medium and in external galaxies. The consistent peak wavelength and profile of the feature suggests that it originates from a carbonaceous solid (Mennella et al. 1998).

The UIE bands consist of vibrational bands of aromatic materials at 3.3, 6.2, 7.7, 8.6, and $11.3 \mu \mathrm{m}$, aliphatic features at 3.4 and $6.9 \mu \mathrm{m}$, unidentified features at 15.8 , $16.4,17.4,17.8$ and $18.9 \mu \mathrm{m}$, as well as broad plateau emission features around 8,12 , and $17 \mu \mathrm{m}$. Although the UIE bands are commonly attributed to polycyclic aromatic hydrocarbon $(\mathrm{PAH})$ molecules, these bands have recently been suggested to be due to mixed aromatic/aliphatic organic nanoparticles (MAON, Kwok \& Zhang 2011, 2013). As much as $20 \%$ of the total energy output in some active galaxies are emitted in the UIE bands, suggesting that organic matter represents a significant fraction of matter in galaxies.

\section{Organic matter in the Solar System}

Although gas-phase hydrocarbons have been detected in Uranus, Neptune, and Titan as early as 1944 (Kuiper 1944), the widespread presence of complex organics in the Solar System was only recognized in the 1960s when paraffins were found in the Orgueil meteorite (Nagy et al. 1961). After the discovery of aromatic and aliphatic compounds in the Murchison meteorite (Cronin et al. 1987), over 14,000 biologically relevant organic compounds have now been identified in the soluble component of carbonaceous meteorites (Schmitt-Kopplin et al. 2010). Over 100 amino acids have been identified, much more 


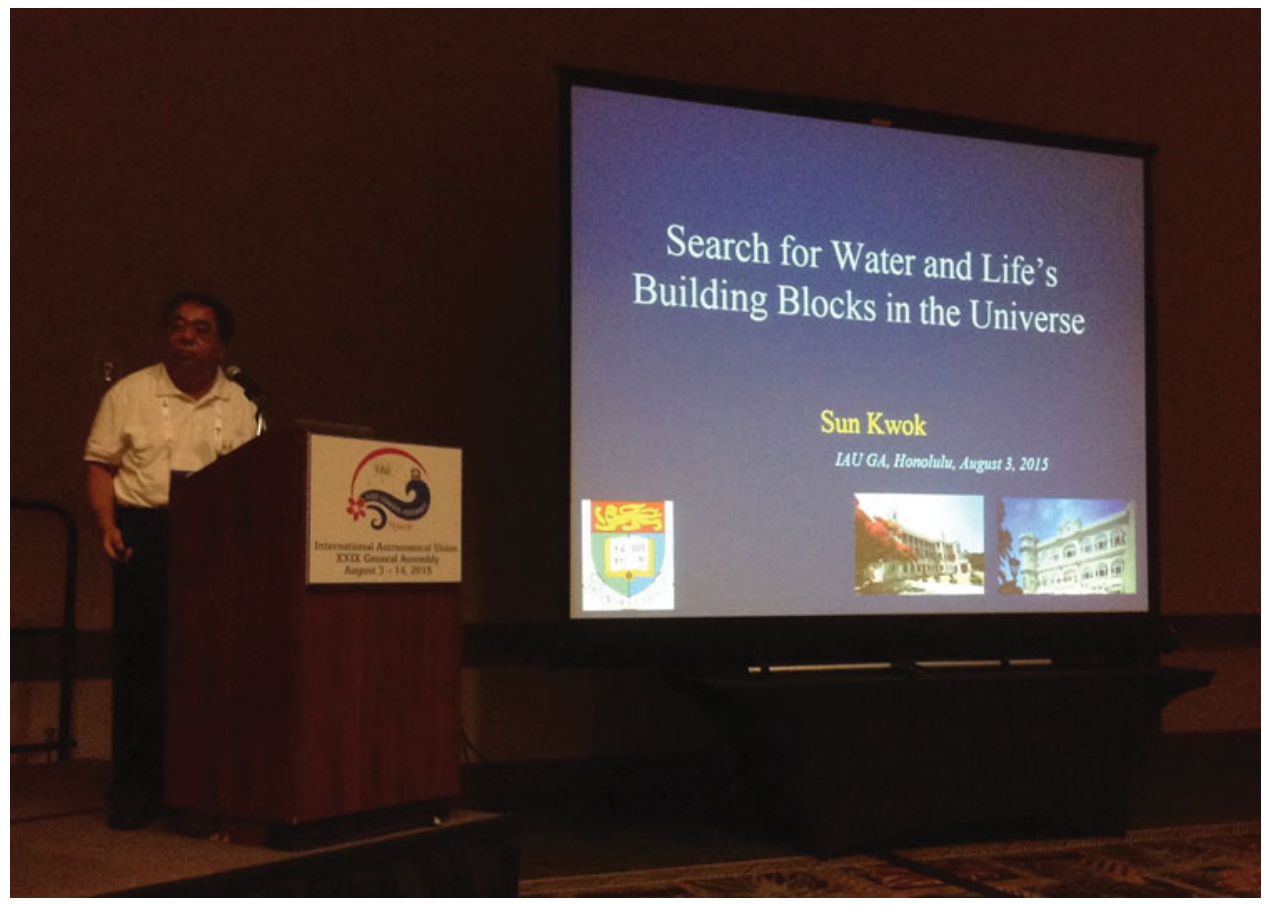

Figure 1. Kwok giving the introductory talk of FM 15.

than the 20 amino acids found in living organisms on Earth. The near equality of D and L chirality of the amino acids suggests that they are not of terrestrial origin. Unusual nucleobases have also been found (Callahan et al. 2011).

Furthermore, $70 \%$ of organic matter in carbonaceous meteorites is in the form of insoluble organic matter, consisting of small (1-4) aromatic rings, short aliphatic chains, and containing heteroelements (O, S, N) (Cody et al. 2011). In terms of abundance, the ratios of the atoms can be expressed as $\mathrm{C}_{100} \mathrm{H}_{46} \mathrm{~N}_{10} \mathrm{O}_{15} \mathrm{~S}_{4.5}$ (Pizzarello \& Shock 2010).

The $3.4 \mu \mathrm{m} \mathrm{C}-\mathrm{H}$ stretching band is found in interplanetary dust particles (IDP) (Flynn et al. 2003). The detection of the $220 \mathrm{~nm}$ feature in IDP suggests that there may be an interstellar-Solar System connection in their respective organic contents (Bradley et al. 1999).

Complex organics in the form of haze is a significant component of the atmosphere of Titan. These nanoparticles are blown into dunes by wind on the surface of Titan. Liquid forms of methane and ethane are also believed to gather on the surface of Titan in the form of lakes. The most popular model for these complex organics is tholins, which are refractory organic materials formed by UV photolysis of mixtures of gaseous nitrogen, methane, and ammonia. Another alternative model is HCN polymer, which is an amorphous hydrogenated carbon nitride formed spontaneously from HCN (Matthews \& Minard 2006).

\section{Conclusions}

With advances in infrared and millimeter-wave observing techniques, water (both in vapor and ice forms) have been detected in interstellar clouds. Water molecules are formed in the outflow of evolved stars. Water ice is commonly present in Solar System objects. What is the connection between circumstellar/interstellar water and solar system ice? 
The reservoir of organic matter on Earth (mostly in the form of kerogen) is the result of life. We now know that complex organic matter of abiological origin is commonly found in Solar System objects. All biologically relevant molecules have been identified in the soluble component of carbonaceous condrites and organic solids of mixed aromaticaliphatic structures are found in meteorites, comets, and on Titan. Recent observational evidence has shown that complex organic matter can be produced in large quantities over short time intervals by stars (Kwok 2004). The key question is: were the Solar System organic substances synthesized in the Solar System in situ or they were brought in from the interstellar medium? To what extent the early Earth was enriched by extraterrestrial organics (Ehrenfreund et al. 2002)?

If complex organics are indeed commonly produced by stars and extensively distributed throughout the Galaxy by stellar winds, will other planetary systems be similarly rich in organics as our own Solar System? If the building blocks of life, water and organics, are commonly available in planets that have habitable environments, would life be easily developed?

\section{Acknowledgement}

This work was partially supported by the Research Grants Council of the Hong Kong Special Administrative Region, China (project no. 17302214).

\section{References}

Bradley, J. P., Keller, L. P., Snow, T. P., Hanner, M. S., Flynn, G. J., Gezo, J. C., Clemett, S. J., Brownlee, D. E., \& Bowey, J. E. 1999, Science, 285, 1716

Callahan, M. P., Smith, K. E., Cleaves, H. J., Ruzicka, J., Stern, J. C., Glavin, D. P., House, C. H., \& Dworkin, J. P. 2011, PNAS 108(34): 13995

Campbell, E. K., Holz, M., Gerlich, D., \& Maier, J. P. 2015, Nature, 523, 322

Cheung, A. C., Rank, D. M., Townes, C. H., Thornton, D. D., \& Welch, W. J. 1969, Nature, 221,626

Cody, G. D., Heying, E., Alexander, C. M. O., Nittler, L. R., Kilcoyne, A. L. D., Sandford, S. A., \& Stroud, R. M. 2011, PNAS, 108, 19171

Cronin, J. R., Pizzarello, S., \& Frye, J. S. 1987, Geochimica et Cosmochimica Acta, 51, 299

Ehrenfreund, P., Irvine, W., Becker, L., Brucato, J. R., Colangeli, L., Derenne, S., Despois, D., Dutrey, A., Fraaije, H., Lazcano, A., Owen, T., \& Robert, F., International Space Science Institute ISSI-Team 2002, Reports on Progress in Physics, 65, 1427

Flynn, G. J., Keller, L. P., Feser, M., Wirick, S., \& Jacobsen, C. 2003, Geochimica et Cosmochimica Acta, 67, 4791

Kuiper, G. P. 1944, ApJ, 100, 378

Kwok, S. 2004, Nature, 430, 985

Kwok, S. 2013, Unexplained spectral phenomena in the ISM, Special Session 16, Highlights of Astronomy, Vol. 16, ed. T. Montmerle, p. 697

Kwok, S. \& Zhang, Y. 2011, Nature, 479, 80

Kwok, S. \& Zhang, Y. 2013, ApJ, 771, 5

Matthews, C. N. \& Minard, R. D. 2006, Faraday Discussions, 133, 393

Mennella, V., Colangeli, L., Bussoletti, E., Palumbo, P., \& Rotundi, A. 1998, ApJ, 507, L177

Nagy, B., Meinschein, W. G., \& Hennessy, D. J. 1961, Annals of the New York Academy of Sciences, 93, 27

Pizzarello, S. \& Shock, E. 2010, Cold Spring Harbor Perspectives in Biology, 2

Schmitt-Kopplin, P., Gabelica, Z., Gougeon, R. D., Fekete, A., Kanawati, B., Harir, M., Gebefuegi, I., Eckel, G., \& Hertkorn, N. 2010, PNAS, 107, 2763

Wallace, L., Bernath, P., Livingston, W., Hinkle, K., Busler, J., Guo, B., \& Zhang, K. 1995, Science, 268, 1155 